\title{
A Sobrevivência em Oncologia: Uma Vivência Paradoxal
}

A partir de um levantamento da literatura especializada, os autores propõem uma revisão crítica da problemática da sobrevivência de pacientes neoplásicos, destacando o caráter paradoxal das vivências dos sujeitos implicados, fornecendo, desta maneira, subsídios para a atuação do psicólogo em serviços de Oncologia.

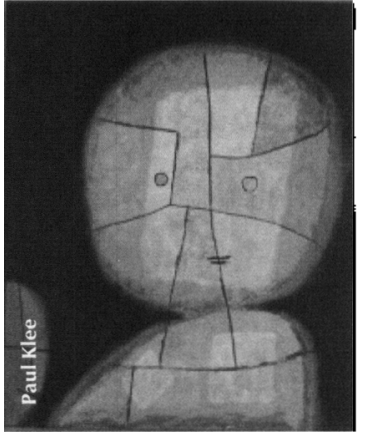

Tereza Cristina Cavalcanti Ferreira de Araujo

Doutoroem Psicologla pela Untwersiodade de Paris $X$ - Nanterre. Professora do instifto de Psicologia do Universidode de Brosilio.

Alessandra da Rocha Arraes

Mestre em Psicologic pela universidade de Brosilio, Psicóloga responsóvel pelo

Serviço de Oncotogic Pedíátrica do Hospital Saran de Brasillo

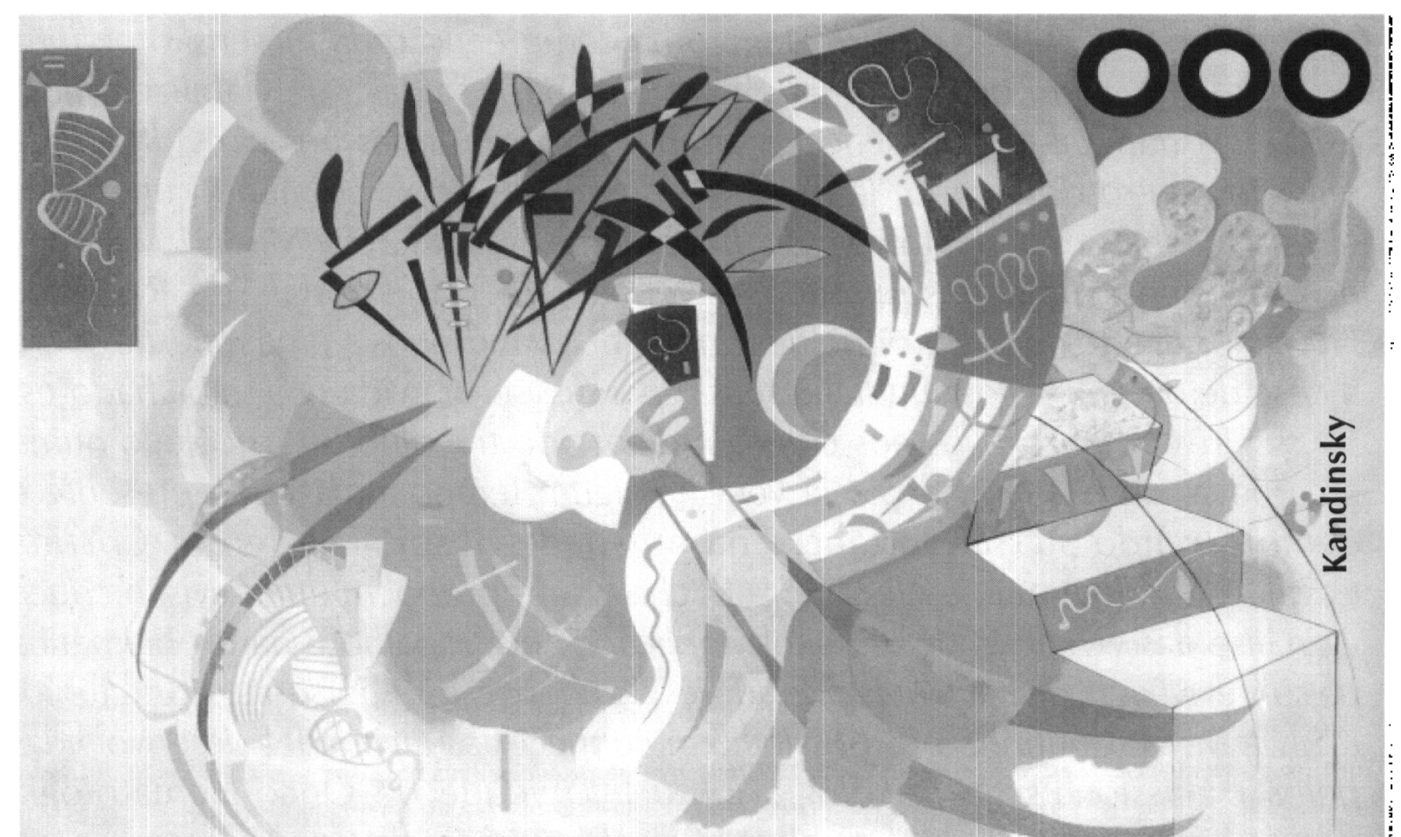

Apesar da intensa e acelerada transferência das conquistas da biotecnologia para a Medicina, ainda são inúmeras as dificuldades pelas quais continuam a passar os portadores de neoplasia no Brasil. Tanto que, de acordo com dados publicados em 1986 pela Divisăo Nacional de Doenças Crônico-Degenerativas do Ministério da Saúde, as neoplasias representariam a causa de 56,1 óbitos em 100.000 habitantes, constituindo a segunda causa mais freqüente de mortalidade nas regiôes Sul e Sudeste. Segundo estimativas feitas pelo Instituto Nacional de Câncer, em 1995, teriam sido diagnosticados cerca de 343.400 novos casos de câncer, sendo que dentre estes poderíamos prever 91.800 óbitos (Borba, 1997). Ou seja, um programa de prevençăo incipiente é freqüentemente seqüenciado por escassos recursos públicos destinados aos centros especializados, por vezes inviabilizando intervençōes diagnósticas e terapêtuticas precoces, corretas e eficazes. Portanto, muitas são as açóes de saúde ainda necessárias no âmbito da prevenção e do tratamento. No entanto, nossa atenção também deve se voltar para aqueles que sobreviveram à experiência oncológica.

Numericamente, eles vêm se tornando mais expressivos. Estima-se que, no ano 2000, um em cada mil jovens adultos, entre 20 e 29 anos de idade, será um sobrevivente de câncer na infância (Varni, Katz, Colegrove \& Dolgin, 1994), o que representaria cerca de 210.000 indivíduos apenas nos Estados Unidos (Lozowski, 1993). No Brasil, desconhecemos indicadores estatísticos semelhantes, mas é possível observar tendências igualmente positivas e encorajadoras, em contraposição à persistência dos problemas anteriormente mencionados. 
Paralelamente, então, ao aumento da taxa de sobrevida para um número cada vez maior de ex-pacientes, surgiram pesquisas voltadas para a temática da sobrevivência em Oncologia.

Desenvolvidas nos anos 60 e 70 , para investigar as seqüelas físicas na sobrevivência - por exemplo, esterilidade, debilidade pulmonar e cardíaca, problemas de crescimento e suscetibilidade para desenvolver novos tumores - passaram, na última década, a ampliar o foco de interesse para os aspectos psicológicos e sociais (Andersen, 1989). Propomos, portanto, uma breve discussão dos principais estudos realizados, enfatizando a aparente contradição dos resultados obtidos em relação às diversas variáveis identificadas nas atuais pesquisas. Sugerimos, ainda, que tais divergências entre os dados são reveladoras do paradoxo que constitui a experiência da sobrevivência.

\section{A Adaptação Psicológica dos Sobreviventes em Oncologia}

Ao analisarmos os trabalhos realizados até o presente momento, podemos constatar a importância atribuída à avaliaçăo da adaptaçăo psicológica dos ex-pacientes neoplásicos. Verificamos, também, que os parâmetros mais estudados envolvem: ansiedade e depressão, nível intelectual e atividade escolar, auto-estima e autoconceito, aspectos conjugais e familiares, relacionamento interpessoal, rede social de apoio, inserção profissional, expectativas para o futuro, distúrbios do comportamento e cuidado excessivo com a saúde. Assim, Koocher, O'Malley, Gogan e Foster (1980), examinando o ajustamento de sobreviventes, encontraram limitação psicossocial, depressão residual, ansiedade e baixa auto-estima. Com a preocupação de identificar variáveis que fundamentem a avaliaçāo da depressão em tais sujeitos, Mulhern, Fairclough, Smith e Douglas (1992) selecionaram fatores de risco - duração de internação, gravidade dos sintomas físicos e depressão materna - e fatores de proteção - status sócio-econômico mais elevado, apoio social para a mãe e competência social da criança. Vale destacar que o melhor preditor da depressão entre estes pequenos pacientes foram os sintomas depressivos das mães medidos pelo Beck Depression Inventory (BDI). No entanto, de modo geral, pesquisas de natureza pluridisciplinar sugerem que não existe comprovaçāo de depressăo mais profunda ou de suicídio entre ex-pacientes de câncer infantil (Tetelbom, Falceto, Gazal, Shansis \& Wolf, 1993). A investigação desenvolvida por Ell, Nishimotto, Morvay, Mantell e Hamovitch (1989) em parte esclarece tais achados contraditórios, uma vez que constata tendência a voltar a estados ansiogênicos e depressivos em momentos de transiçăo dentro do ciclo de desenvolvimento, como, por exemplo, no casamento e na inserção da vida profissional.

Em suma, ao tentar responder se os sobreviventes oncológicos seriam mais ou menos ansiosos e mais ou menos depressivos, a literatura especializada tem se apresentado insatisfatória. Ao que tudo indica, às dificuldades próprias da análise da sobrevivência somam-se aqui aquelas da avaliação e mensuração da ansiedade e depressão (Eiser \& Havermans, 1994), especialmente quando se refere a pacientes pediátricos (Mulhern, Fairclough, Smith \& Douglas, 1992). Gouveia, Barbosa, Almeida e Gaiāo (1995) mostram-se, entretanto, mais otimistas e sugerem o uso do Child Depression Inventory (CDI), versăo infantil do $\mathrm{BDI}$, do qual sāo responsáveis pela adaptação no Brasil.

Além da ansiedade e depressão, outras dificuldades de ordem emocional e afetiva têm merecido interesse dos autores. Frisch e Desmarez (1988) identificaram problemas como: desvalorização da imagem corporal, baixa auto-estima, fragilidade narcísica e perturbação da orientação temporal. Tomando por base entrevistas psicoterápicas com cerca de cem sujeitos curados no Instituto Gustave-Roussy em Paris (alguns vinte anos após o encerramento do tratamento), Oppenheim (1996) constata que "a violência da experiência subjetiva atravessada não se atenua com o tempo" (p. 285). Este sofrimento pode, por exemplo, se expressar em condutas de risco, por vezes autodestrutivas. Assim, quando abordados mais diretamente, indivíduos com situações escolares, sociais, familiares e profissionais satisfatórias, acabam por revelar um grande desamparo, testemunhando a não superação das dificuldades. "a violência do experiência subjetiva atrovessada nõo se atenua com o tempo" Oppenheim (1996) 
Os resultados levantados por Greenberg, Kazak e Meadows (1989) identificaram um autoconceito significativamente mais pobre entre os sobreviventes do que entre os sujeitos do grupo-controle. $\mathrm{E}$, dentre os sobreviventes, aqueles mais gravemente atingidos por seqüelas físicas obtiveram escores mais baixos do que os menos atingidos. Os sobreviventes também apresentaram mais loci de controle externo do que os demais, apesar de ambos os grupos situarem-se dentro das médias normativas. $\mathrm{Na}$ revisăo proposta por Zelter (1992), não se verificaram maiores distúrbios psicológicos ou psiquiátricos entre os sobreviventes, na medida em que eles apresentaram os mesmos fatores de risco que a populaçăo em geral.

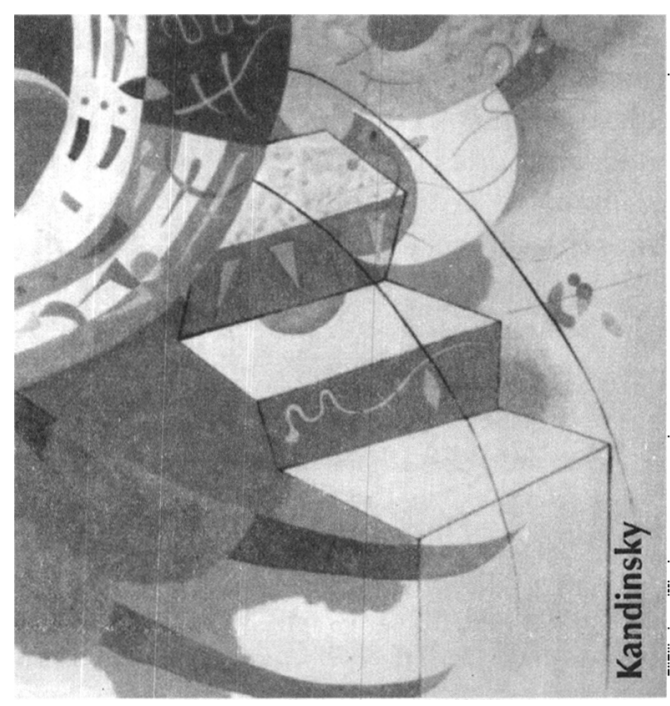

Foi possível constatar, entretanto, que existem dificuldades de ajustamento psicológico, principalmente no que se refere à preocupação excessiva com a saúde. Tal preocupação se concretizaria, por exemplo, pela procura de serviços de urgência hospitalar e por uma vigilância constante diante do aparecimento de sintomas físicos, com intempestiva solicitação de exames médicos. Segundo Carpenter, Morrow e Schmale (1989), com o passar do tempo, isto se minimizaria, ou seja, um grupo de sobreviventes mais antigos demonstraria menos excesso de cuidado com a saúde que um grupo mais recentemente curado. Já Makiperneaa (1989) encontrou resultados divergentes, na medida em que aproximadamente metade dos sobreviventes da sua amostra diziam se sentir em excelentes condiçōes de saúde, enquanto a outra metade continuava a reportar tais problemas, bem como dificuldades cotidianas em geral. Greenberg, Kazak e Meadows (1989) chegaram a constataçöes semelhantes, uma vez que os sobreviventes apresentaram escores similares aos do grupo-controle em relaçăo à preocupaçāo com a própria saúde. Em ambos os grupos, mais da metade dos sujeitos afirmaram nâo temerem pela condiçăo física. Cerca de um terço de indivíduos dos dois grupos acreditavam poder contrair uma doença grave. Em compensação, $10 \%$ dos sobreviventes tinham medo da recidiva do tumor, enquanto que somente $1 \%$ do grupo-controle tinha medo de ser acometido de câncer.

Outra dimensāo da adaptação freqüentemente avaliada pelos autores $e ́ \mathrm{a}$ inserção escolar. Deve-se lembrar que as intercorrências provenientes da doença e do tratamento, associadas a uma rotina de atendimento que ainda depende de internaçóes (em detrimento do esquema de assistência ambulatorial), além da flagrante carência de programas hospitalares para acompanhamento escolar, provocam o adiamento ou a interrupção da escolarização. Os trabalhos de Greenberg e Meadows (1991), Zelter (1992), Faulkner, Peace e O'Keeffe (1995) apontam tais prejuízos. Entretanto, Evans e Radford (1995) não encontraram diferenças entre o nível educacional dos sobreviventes e de um grupo-controle, apesar do maior interesse e disposiçāo dos sujeitos do grupo-controle para seguir estudos universitários em comparaçăo com os ex-pacientes oncológicos. Gray et al. (1992) descobriram maior tendência a repetência, fracasso e evasão escolar, mas a exemplo da pesquisa anterior, puderam notar que nos níveis mais avançados de escolaridade as diferenças não mais existiam. Ao que parece, com o passar dos anos, o sobrevivente acabaria por alcançar seus pares. Chamam atenção, ainda, as expectativas de pais e professores em relação ao desempenho acadêmico dos sobreviventes. De acordo com Valle (1994a), os pais explicariam as dificuldades escolares não pela baixa.assiduidade às aulas, mas pelas seqüelas físicas provocadas pela doença e pelo tratamento, mantendo-se assim em conformidade exclusivamente com a perspectiva médica. Já os 
professores parecem carecer de orientação adequada por parte das instituiçōes de saúde, uma vez que suas opiniôes revelam concepções errôneas e preconceituosas em relação a seus alunos que tiveram câncer. As pesquisas mostram que os mestres tendem a avaliá-los negativamente, atribuindo-lhes dificuldades de concentração e de socialização. Sob o ponto de vista dos professores, as desordens comportamentais inclusive se agravariam com o decorrer do tempo, tornando tais diferenças ainda mais açentuadas (Davies, 1992).

E quanto ao futuro, será que os sobreviventes têm expectativas e projetos? Quais são eles? Ou, a condição mesmo de sobrevivente implicaria viver apenas no tempo presente? Para diversos pesquisadores, a capacidade de se projetar além do aqui e agora é considerada como variável preditora da qualidade da sobrevivência, e o que se tem justamente evidenciado é uma dificuldade em planejar e buscar o futuro entre os sobreviventes (Stalker, Johnson \& Cimma, 1989; Lozowski, 1993; e Oppenheim, 1996). Isto se expressa, por exemplo, na tendência a adiar decisōes significativas para o ciclo do desenvolvimento, tal como o casamento ou a escolha profissional. Todavia, o estudo de Byrne et al. (1989) não verificou tais indícios.

Apesar de focalizadas na sua maior parte em aspectos negativos da sobrevivência, as pesquisas têm assinalado também que para os sujeitos assim implicados, a superação da experiência oncológica pode propiciar mudanças positivas e crescimento pessoal. Desta maneira, os entrevistados de Lozowski (1993) disseram ter adquirido maturidade reconhecendo mais claramente o propósito de suas vidas. Gray et al. (1992) também verificaram vantagens adaptativas (maior controle emocional e maior motivação) para os sobreviventes de câncer pediátrico em relação a seus pares.

O valor clínico das conclusōes obtidas por cada uma dessas investigaçōes é inegável, todavia somos confrontados com a flagrante contradição entre os resultados. Em parte, tal dificuldade pode ser compreendida pelo fato de que a questão da cura e da sobrevivência não é de abordagem simples, sobretudo em um contexto de interface como o da Psiconcologia. Na realidade, superpóem-se nesta problemática as noções da cura física, no plano estritamente médico, e da cura, em seu sentido mais amplo, abrangendo as dimensões psicológicas e sociais do sujeito. Mais do que isso, verificamos que mesmo os critérios para o estabelecimento da cura orgânica não são consensuais entre os autores. Para citar apenas alguns, para Andersen (1994) e para Eiser e Havermans (1994), o indivíduo é considerado curado somente quando não apresenta sintomas há pelo menos cinco anos. Carpenter, Morrow e Schmale (1989) estabelecem o período de dois anos fora de tratamento, e Garre (1994) o fixou em dois anos e meio. Ora, a diversidade de critérios na área médica incide obviamente

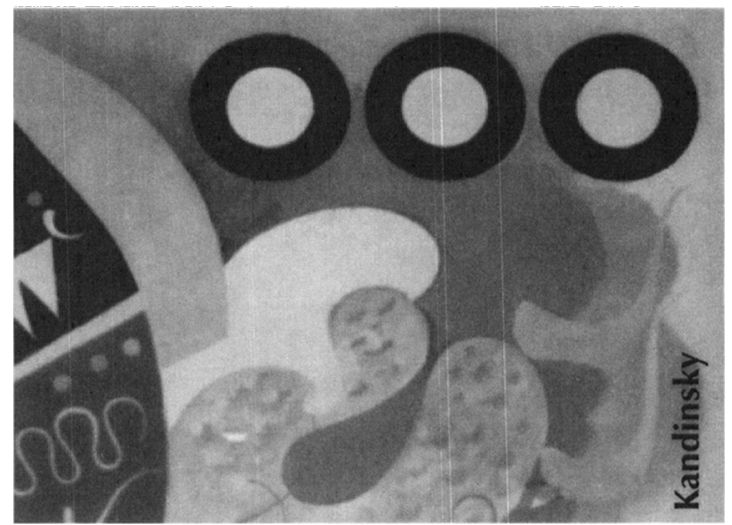

sobre as investigaçōes desenvolvidas na esfera da Psiconcologia. Tanto que os critérios de sobrevivência variam de seis meses para Valle (1994a), Speechely e Noh (1992), Carpenter, Morrow e Schmale (1989) até cinco anos para Marcken, Gailly, Brichard, Vermylen, Ninane e Cornu (1996), Evans e Radford (1995), Greenberg e Meadows (1991), Kazak e Meadows (1989) e Byrne et al. (1989). Existem mesmo aqueles que nāo delimitam qualquer critério temporal no delineamento de pesquisa, como Lozowski (1993), Kant, Glover, Horm, Schatzken e Harris (1992), Davies (1992), Keith (1991), Rosenthal, Fraissinet, Lemaitre, Aussilloux $\mathrm{e}$ Visier (1988), considerando suficiente que os sujeitos tenham encerrado o tratamento.

A grande heterogeneidade das amostras constitui outra restrição metodológica. Incluem-se em um mesmo estudo, sujeitos com diferentes idades abarcando uma grande amplitude 
de faixas de desenvolvimento. Indistintamente săo comparados entre si, casos de sobrevivência a diferentes tipos de tumores com estágios de gravidade e condutas terapêuticas diferenciadas. Evidentemente tal disparidade ou ausência de critérios dificulta a comparação entre os trabalhos, limitando possíveis generalizaçōes sobre importante questão.

\section{O Sobrevivente e sua Família}

Viver com câncer pode ser considerado uma das experiências mais estressantes pela qual uma pessoa e sua família podem passar (Kazak \& Meadows, 1989; Faulkner, Peace \& O'Keeffe, 1995), especialmente quando o doente é uma criança. A literatura tem esclarecido sobre o enfrentamento familiar em situaçōes de ruptura como aquelas provocadas pelo diagnóstico de câncer, a seqüência de intervençöes terapêuticas e mesmo pela morte. Mas muito pouco se conhece sobre como estão as famílias daqueles que sobreviveram a um câncer na infância ou em outras etapas do desenvolvimento.

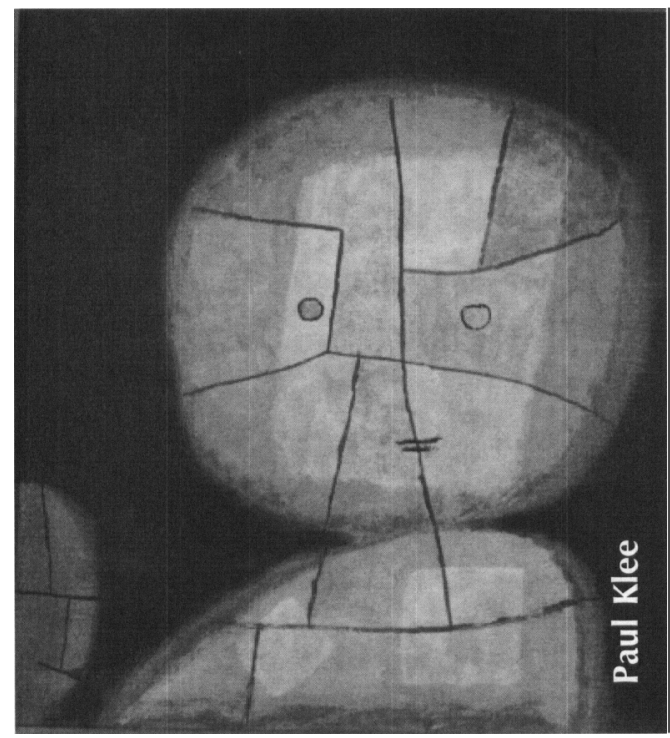

As fases do diagnóstico, da alta e da primeira recidiva têm sido apontadas pela literatura como as mais difíceis para a família do sobrevivente (Pucheu, 1988; Valle, 1994b; Ribeiro, 1994; Faulkner, Peace \& O'Keeffe, 1995). Muitos pais sentem-se deprimidos e a dinâmica familiar sofre profundas reformulações com a redefinição de papéis e funçōes (Minuchin, 1987; Gotay, 1987; Tetelbom et al., 1993).
Problemas conjugais começam a surgir em razão das grandes solicitações de ajustamento individual e grupal. Lansky, Cairns, Hassamein, Wehr e Lowman (1978) verificaram altos niveis de estresse conjugal, mas a taxa de divórcio entre os pais dos sobreviventes mantinha-se na média da população geral. $\mathrm{O}$ ajustamento marital dos pais de crianças diagnosticadas com câncer foi investigaclo por Dahlquist et al. (1996), ao realizar um estudo longitudinal, no qual se avaliou o estudo de enfrentamento e as respostas afetivas em dois momentos: dois e vinte meses após estabelecido o diagnóstico. $\mathrm{O}$ nivel de ansiedade das mães diminuiu entre a primeira e a segunda avaliação, entretanto os pais năo tiveram a mesma evolução. $\mathrm{O}_{5}$ autores concluíram ainda que o estado de saúde da criança é preditor em relação ao casamento para os pais, mas não para as mães. De modo geral, o ajustamento marital não se alterou ao longo do tempo. Contraditoriamente, dos vinte e quatro casais avaliados por Greenberg e Meadows (1991) vinte e um afirmaram ter desenvolvido um melhor entrosamento no casamento.

Alguns autores têm proposto a avaliaçāo do Transtorno de Estresse Pós-Traumático (PTSD) no intuito de avaliar a adaptaçăo dos familiares de sobreviventes. Ao comparar a prevalência de PTSD em mães de sobreviventes com mães de crianças fisicamente saudáveis, Pelcovitz et al. (1996) notaram que nas primeiras foram diagnosticados sintomas de estresse pós-traumático. Os autores concluíram que a "maternagem" de uma criança com câncer comporta elementos considerados como de alto risco para PTSD: ameaça vital, hospitalização, incertezas, seqüelas físicas, baixa controlabilidade, medo extremo e falta de preparo anterior. Em outro estudo comparativo entre sobreviventes de câncer pediátrico e sujeitos que não vivenciaram doenças crônicas, e pais de ambos os grupos, Kazak et al. (1997) encontraram resultados semelhantes. Pais e mães dos sobreviventes igualmente relataram elementos significativos de estresse pós-traumático, como, por exemplo, insistentes flashbacks sobre o câncer e o período de tratamento. Em compensação, quanto ao relato de sintomas, os sobreviventes não diferiram do grupo-controle. Adotando procedimentos semelhantes 
aos anteriores, Stuber, Christakis, Houskamp e Kazak (1996) verificaram que $12,5 \%$ dos sobreviventes, $33,3 \%$ dos pais, $39,7 \%$ das mães em sua amostra apresentaram sintomas reveladores de estresse pós-traumático (ansiedade exacerbada e reminiscências) até doze anos após o término do tratamento. Os escores das mães e dos pais são significativamente correlacionados, mas somente os escores das mães estão correlacionados aos dos filhos.

Sem dúvida, é fundamental que se realizem mais pesquisas sobre tal dimensão da adaptação e enfrentamento familiares em condiçōes de sobrevivência, sobretudo através de estudos longitudinais.

\section{A Sobrevivência é uma Vivência Paradoxal}

É importante destacar que a percepção de si mesmo como curado não se dá prontamente para o sobrevivente. Ao ser comunicado da provável erradicação do tumor, inicia-se a fase de manutenção que marcará o início de uma longa batalha contra os riscos de uma recidiva. Vale lembrar que ainda por muitos anos existirá uma maior probabilidade para o sobrevivente, mais do que para outro indivíduo da população em geral, de desenvolver uma neoplasia idêntica à primeira ou de outro tipo histológico (Nicholson, Fears \& Byrne, 1994).

Inicia-se, assim, um prolongado confronto com os constantes paradoxos que marcarão as etapas seguintes.

Os sobreviventes podem então reagir à notícia da alta com ambivalência. Por um lado, podem se sentir aliviados e eufóricos com o término do tratamento. Por outro lado, sentem-se inseguros sem a proteção do ambiente hospitalar e o suporte medicamentoso. Podem perceber-se como vulneráveis. A isto somam-se as dúvidas quanto à durabilidade da recuperaçào e quanto às dificuldades relacionadas à retomada da vida cotidiana com todas as adaptações que ainda podem ser necessárias. Em outras palavras, o sobrevivente está curado, mas tem mais chances de se tornar um doente. Não precisa mais de quimioterapia, mas o acompanhamento mé- dico é indispensável e alguns medicamentos ainda podem ser prescritos, até porque podem existir seqüelas. (Alby, 1988; Alapetite, Baillet, Champemond, Dessard-Diana, Housset \& Michel-Langlet, 1988; Valle, 1994a; e Faulkner, Peace \& O'Keeffe, 1995).

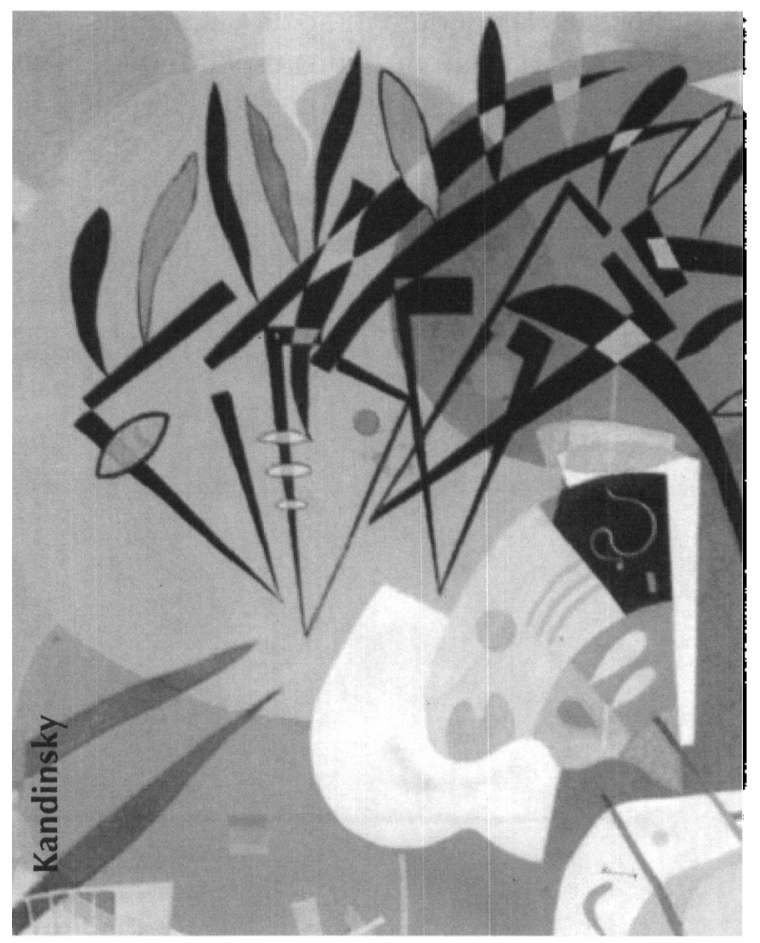

Portanto, a integração da noção de cura não é evidente para o sobrevivente, o que pode ser constatado na prática do profissional de saúde atuando em Oncologia, especialmente o psicólogo da unidade. Consideramos, também, que as aparentes contradições entre as investigações e, às vezes, em uma mesma pesquisa, são inerentes à questão da sobrevivencia. Elas seriam provocadas pelas repercussōes da vivência paradoxal que caracteriza tal condição. Desta maneira, parecenos indispensável estabelecer como objetivo do acompanhamento psicológico, tanto para o paciente em expectativa de alta como também para o sobrevivente, uma avaliação dos recursos internos e externos para manejar com o paradoxo inerente à condição da sobrevivência. Identificadas as insuficiências nesse plano, é meta da conduta terapêutica orientar para o desenvolvimento de tais potencialidades do sujeito, evitando a sua alienação e paralisação em um periodo dramático da sua vida. 


\section{Consideraçöes finais}

Permanece, portanto, a indagaçảo quanto à sobrevivência em Oncologia. Acreditamos que estudos nesta área venham a constituir contribuições para oferecer acompanhamento psicológico aos sobreviventes e a suas famílias. Compreender por que alguns sobrevivem e outros não, por que alguns sobrevivem bem e outros não, sem dúvida favorece também a adoção de estratégias preventivas mais adequadas às necessidades de nossos pacientes desde as fases iniciais da conduta terapêutica. $\mathbf{E}$, mais do que isso, pensamos que estudar a sobrevivência em Oncologia ou em outras especialidades médicas, permitirá conhecer melhor a sobrevivência em outras "situações-limite" da existência humana.

Tereza Cristina Cavalcanti Ferreira de Araujo
Alessandra da Rocha Arraes
instituto de Psicologia, UnB, 70910-900, Brasfia, DF.
E-mail: araujotc(Gguarany,unb.br
Fax+(06t) 349-0183 - Fone; (061) 348-2624.

Alapetite, C., Baillet, F., Champemond, S., Dessard-Diana, B., Housset, M\& Michel-Langlet, P. (1989). Opposition pour le malade cancereux

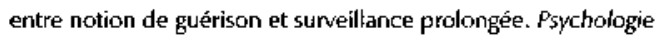
Médicale, 20 (9), 1283-1284.

Alby, N, \{ 1988\}. Dif́iculté pour les parents d’intégrer la notion de guérison: conséquences à long terme de la makadie sur les familles. Psychologie Médicale, 20 (9), 1350-1351.

Andersen, B. L. (1989). Directions for a psychology research agenda in cancer. Health Psychology, 8 (6), 753-762.

Andersen, B. L. (1994). Surviving cancer, Cancer supplement, 74 (4), $1350-1493$

Borba, S. R. C. (1997). Dificuldades para o tratamento da dor do cancer no Brasil, Em C. M. Nascimento-Sctulze (Org.), Dimensòes da dor no cáncer, Reflexōes sobre o cuidado interdisciplinar e um nowo paradigma da saúde (p.135-156). Sâo Paulo: Robe Editorial.

Byrne, J. Fears, T. R., Steinhom, S. C., Mulvihill, I. J., Connelhy, R.R., Austin, D. E. Holmes, G. E. Holmes, F. F., Latourette, H. B., Teta, M. I. Strong, L. C., \& Myers,. H. (1989). Marriage and divorce after chikdhood and adolescent cancer. Journal of the American Medical Association, 262 (19), 2693-2699.

Carpenter, P. J., Morrow, G. R., \& Schmale, A. H. (1989). The psychosocial status of cancer patients after cessation of treatment. fournat of Psychosocial Oncology, 7 (12), 95-103.
Dahlquist, L. M., Czyzewski, D. I. \& Jones, C. L. (1996). Parents of children with cancer: a longitudinal study of emotional distress, coping style, and marital adjustement two and tweenty months after diagnorsis. fournal of Pediatric Psychology, 21 (4), 541-554.

Davies, D. (1992). Psychotherapy of a preschool cancer survivor: promoting mastery and understanding. Child and Adolescent Social Work koumal, 9 (4), 289-305

Ell, K, Nishimolto, R, Mkirvay, T., Mantell, J. \& Hamositch, M. (1989). A longitudinal analysis of psychological adaptation amongsurvivors of cancer. Cancer, 63 (2), 406-413.

Eiser, C. \& Havemans, T. (1994). Long term social adjustment after treatment for childhoud cancer. Archives of Disease in Childhood, $70(1), 66-70$.

Evans, S. E. \& Radford, M. (1995). Current lifestyle of young adults treated for cancer in childhood. Archives of Disease in Childhood, 72 15), $423-426$

Faulkner, A, Peace, G. \& O Keeffe, C. (1995). When a child has cancer London: Chapman \& Hall.

Frisch, G. A. \& Desmarez C. (1988). Séquelles psychologiques et cancerchez l'enfant en rémission. Psychologre Médicale, 20 (9), 1343 1344. 
Garre (1994). Health status of long-term survivors after cancer in childhood. journal of Pediarric Hematology and Oncology, 76 (2). 143-152.

Gray, R. E, Duan, B. D., Shermer, P., Fitggerald, A. V, Berry, M. P. Jenkin, D. \& Doherty, M. A. (1992). Psychologic adaptation of survivors of childhood cancer. Cancer, 70 (11), 2713-2721.

Greenberg, H. S., Kazak, A. E., \& Meadows, A. T. (1989). Psychologic

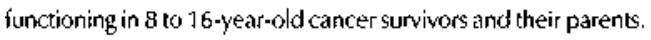
The journal of Pediatrics, 174 (3), 488-493.

Greenberg, H. S. \& Meadows, A. T. (1991). Psychosocial impact of cancer survival on school-age children and their parents. Journal of Psychosocial Oncology, 9(4), 43-55.

Gotay, C. C. (1987). Quality of life amongsurvivors of hildhood cancer: a critical review and implications for intervention. Journal of Psychosocial Oncology, 5 (4), 5-21.

Couveia, V. V., Barbosia, C. A., Almeida, H. J. F, \& Caiāo, A. A. (1995). Inventário de Depressảo Inf́antil - CDI: estudo de adaptaçảo com escolares de loz̄o Pessoa, jornal Brasileiro de Psiciuatria, 44 (7), 345 349.

Kant, A. K., Clover, C., Horm, J., Schatzken, A., \& Harris, T. B. (1992). Does cancer survival difier for okder patients? Cancer, $70(11), 2734-$ 2740 .

Kazak, A. E., \& Meadows, A. T. (1989). Families of young adolescents who have survived cancer: social-emocional adjustment, adaptability, and social support. fournal of Pediatrics P5ychology, 14 (2), 175-191.

Kazak, A. E., Meeske, K, Penati, B., Barakat, L. P., Christakis, D., Meadows, A. T., Casey, R. \& Stuber, M. L. (1997). Posttraumatic stress, family functioning, and social support in survivors of childhood leukemia and their mothers and fathers, Journal of Consulting and Clinical Psychology, 65 (1), 120-129.

Keith, S. J. (7991\}. Surviving survivorship: creating a balance, fournal of Psychosocial Oncology, 9 (3), 109-115.

Koocher, G., OMalley, ). E., Gogan, J. L., \& Foster, D. (1980). Psychological adjustment among pediatric cancer survivors, fournat of Child Psychology and Pychiatry, 27 (2), 163-173.

Lansky, S. B., Caims, N. U., Hassannein, R., Wehr, \}, \& Lomman, T. (1978). Childhood cancer: parental discord and divorce. Pediatrics, 2, 184 188.

Mulhern, R.K., Fairclough, D.L., Smith, B. \& Douglas, S.M. (1992). Maternal depression, assessment methods, and physical symptons affect estimates of depressive symptomatology among children with cancer.Journal of Pediatric Psychology, 17 (3), 313. 326.
Nichokson, H. S., Fears, T., \& Byrne, J. (1994). Death during adulthood in survivors of childhood and adolescent cancer. Cancer, 73 (12), 3094-3102

Oppenheim, D. (1996). Devenir psychologique des eniants guéris d'une affection cancéreluse. Neuropsychiatrie de f'Enfance et de I'Adolescence, 44 (6-7), 285-294.

Pelcovitz D., Goldenberg, B., Kapken, S., Weinblatt, M., Mandel, F., Meyers, B. \& Vinciguena, V. (1996). Posttraumatic stress disorder in mothers of pediatric cancer survivors. Psychosomatics, 37 (2), 116126.

Pucheu. S. (1988). Le sentiment de guérison et ses aléas psychiques. Psychologie Médicale, 20 (9), 1279-1280.

Ribeiro, E. M. P.C. (1994). O paxiente terminal e a familia. Em M. M. J. Carvalhoet at. Introdtuçāo à psiconcologia (p. 197-218). Campinas: Editorial Psy II.

Rosenthal, C; Fraissinet, M.; Lemaitre, L.; Aussilloux, C. \& Visier, J. P. (1988). Evolution de I'enfant cancéreux versus guérison: aspects psychologiques et sogiaux. Psychologie Médicale, 20 (9), 1345-1346.

Speedhley, K. N. \& Noh, \$. (1992). Surviving childhood cancer, social support, and parents' psychological adjustment Journal of Pediatric Psychology, 17 (1), 15-31.

Stakker, M, Z., Johnson, P. S. \& Cimma, C. (1989). Supportive activities requested by survivors of cancer. Journal of Psychosocial Oncology, 7 (4), $21-31$.

Stuber, M., Christakis, D., Houskamp, B. \& Kazak, A (1996). Posttauma symptoms in childhood leukemia survivors and their parents. Psychosomatics, 37 \{3\}, 254-261.

Tetelbom, M., Falceto, O. C., Gazal, C. H., Shansis, F, \& Wolf, A. L. (1993). A criança com doença crônica e sua familia: importància da avaliaçáo psicossocial. fornal de Pediatria, 69 (1), 5-11.

Valle, E. R. M. \{1994at. Algumas conseqüências psicossociais en crianças curadas de căncer - visão dos pais, Jornal de Pediacria, 70 (1) 27.27 .

Valle, E. R. M. (1994b). Vivêncías da familia da criança com câncer. Em M. M. I. Calvalho et at. introduçāo a psiconcologia (p. 219-242). Campinas: Editorial Psy II.

Vami, f. W. Katz, E. R., Colegrove, R. \& Dolgin, M. (1994). Perceived social support and adjustment of children with newly diagnosed cancer. journal of Developmental and Behavioral Pediatrics, 15 (1), 20-26.

Zelter, L. K. (1992). Cancer in adolescents and young adults psychosocial aspects. Cancer Supplement, 가 \{10\}, 3462-3467. 